\title{
Perceptual localization of visual stimuli flashed during saccades
}

\author{
HITOSHI HONDA \\ Niigata University, Niigata, Japan
}

\begin{abstract}
Subjects were asked to make a saccade to a visual target flashed in the dark during a prior primary saccade, and to report its apparent position by moving an adjustable light spot to that position. When targets were presented at the beginning of the primary saccade, subjects perceptually mislocated them in the direction of the saccade, whereas when targets were presented immediately before the end of the primary saccade, the flashed targets were mislocated in the opposite direction. The perceptually localized position of the target was primarily determined by its retinal position. However, at all actual and retinal positions of the target, the localized position shifted from the position that would be predicted if the location of the target was determined only by its retinal position to the prior primary sacade direction. The results were discussed in relation to extraretinal eye position signals. Subjects moved their eyes not to the actual position of the target, but to its apparent position. In some trials, there was a discrepancy between perceptual and oculomotor localization, which was interpreted as having been caused by the imprecise localization ability of the oculomotor system.
\end{abstract}

The first aim of this study was to examine the accuracy of perceptual localization of a visual stimulus briefly presented in the dark. Such localization has been extensively studied by Matin and his colleagues (Matin, Matin, \& Pearce, 1969; Matin, Matin, \& Pola, 1970; Matin \& Pearce, 1965). They asked their subjects to report whether a test flash that occurred sometime before, during, or after a saccade lay to the left or the right of a previously seen fixation target, and measured the point of subjective equality of the fixation target near the time of a saccade. They showed that errors in perceptual localization occurred before the saccade onset and finished slightly after it. Similar results have been shown when visual targets were presented on an illuminated steady background (Bischof \& Kramer, 1968; Mateeff, 1978). This study examined the details of the time course of perceptual mislocalization when saccades were evoked by stimuli presented against a dark background.

In Matin's studies (Matin et al., 1969; Matin et al., 1970; Matin \& Pearce, 1965), perceptual localization was examined at the position of the original fixation point. However, there is a possibility that localization may differ for different actual positions of the target in space and for different retinal positions. Therefore, I measured the localization of targets by presenting them at nine different positions in space, and analyzed the localized position in relation to both actual and retinal positions of the target.

This research was in part supported by a 1985 Grant-in-Aid for Scientific Research (No. 61510038) from the Japanese Ministry of Education, Science and Culture to the author. Requests for reprints should be sent to Hitoshi Honda, Department of Psychology, Faculty of Humanities, Niigata University, Ikarashi, Niigata-shi, Niigata 950-21, Japan.
The second aim of this study was to compare perceptual and oculomotor localizations during a saccade. Many studies have suggested that there is a substantial discrepancy between motor and perceptual localization near the time of eye movements (Bridgeman, Lewis, Heit, \& Nagle, 1979; Hansen, 1979; Hansen \& Skavenski, 1977; Honda, 1985; Mitrani, Dimitrov, Yakimoff, \& Mateeff, 1979). Hansen and Skavenski (1985) reported that subjects accurately struck with their unobservable hand targets flashed at various times before, during, or after saccadic eye movements. This accuracy contrasts with the high degree of mislocalization shown in perceptual judgment experiments. In the present study, I presented the first visual target to the right of the original fixation point and asked the subjects to make a saccade toward it. During the primary saccade, a second visual target was flashed. The subjects were asked to rapidly make a secondary saccade to where the second visual target had appeared (oculomotor localization). In addition, the subjects were asked to report the apparent position of the second target by moving a small light spot to that position (perceptual localization). We compared the accuracy of these two kinds of localization tasks.

The problem of oculomotor localization of visual stimuli presented during a saccade is very important in relation to the theory of the mechanism of saccade generation. Hallett and Lightstone (1976a) showed that their subjects could direct their eyes to brief visual stimuli triggered by prior saccades. Their results were interpreted as evidence that retinal image distance from the fovea is not a critical error signal that determines the size and the direction of saccades. They concluded that the interaction of retinal and eye position information is such that saccades are goal directed. We attempted to obtain additional evidence for this idea. 


\section{METHOD}

\section{Apparatus and Procedure}

A subject was seated in a dark room with his/her head fixed by a dental bite board and a forehead rest. The subject's horizontal eye movements of the right eye were monitored by means of a photoelectric apparatus with a pair of phototransistors (Toshiba TPS 601) and an infrared light-emitting diode (LED; Toshiba TLN 101). After careful adjustment of the position of the phototransistors, the accuracy was to $0.5^{\circ}$ of arc, with a resolution of better than $0.2^{\circ}$ of arc.

On each trial, a buzzer warning signal ( 1 sec in duration) was given, and then a fixation point (red LED with a diameter of $0.2^{\circ}$, $20 \mathrm{~cd} / \mathrm{m}^{2}$ ) was presented at the center of the visual field. The distance from the subject's eyes to the fixation point was approximately $57 \mathrm{~cm}$. The subject was asked to watch the fixation point. Two milliseconds after the offset of the fixation point, a visual stimulus for saccades was presented for $20 \mathrm{msec}, 8^{\circ}$ to the right of the fixation point. The subject was asked to make a saccade (Saccade $S 1$ ) to the visual stimulus (Target $\mathrm{T} 1$ ). Target $\mathrm{T} 1$ consisted of two vertically arranged rectangular red LEDs (Figure 1). Each LED $\left(10 \mathrm{~cd} / \mathrm{m}^{2}\right)$ was $0.1^{\circ}$ of arc in height and $0.3^{\circ}$ in width. The distance between the centers of the LEDs was $0.4^{\circ}$. To avoid anticipatory saccades, we employed five different durations for the original fixation point: $1.0,1.2,1.4,1.6$, and $1.8 \mathrm{sec}$. When the subject made a saccade either before the presentation of Target $\mathrm{T} 1$ or $400 \mathrm{msec}$ after the offset of the fixation point, Target $T 2$ was never presented.

During a saccade, the second visual stimulus (Target $\mathrm{T} 2$ ) for perceptual and oculomotor localization was presented for $2 \mathrm{msec}$. To present Target $\mathrm{T} 2$ during a saccade, the output of the eye movement monitor was fed to a window discriminator, which triggered the onset of Target $T 2$. The position of Target $T 2$ varied from trial to trial. Nine different positions were employed: $-4^{\circ},-2^{\circ}, 0^{\circ}$, $+2^{\circ},+4^{\circ},+6^{\circ},+8^{\circ},+10^{\circ}$, and $+12^{\circ}$ of arc, where the minus and the plus signs indicate the left and the right sides, respectively, of the fixation point. The diameter of Target $\mathrm{T} 2$ was $0.3^{\circ}$ of arc, and the luminance level was $40 \mathrm{~cd} / \mathrm{m}^{2}$. The subject was asked to move his/her eyes (Saccade S2) as rapidly as possible to where Target T2 had appeared, and to maintain fixation. When the subject could not detect Target $T 2$, he/she reported it by pressing a key.

One point four seconds $(1.4 \mathrm{sec})$ after the offset of Saccade S2, a small red LED was presented for $6 \sec \left(0.2^{\circ}\right.$ of arc in diameter, $20 \mathrm{~cd} / \mathrm{m}^{2}$ ). The subject could manipulate the horizontal position of this stimulus in $1^{\circ}$ steps by rotating a knob. The subject was asked to indicate the apparent position of Target $\mathrm{T} 2$ by moving the red LED to that position. During localization, the subject was allowed to move his/her eyes freely. In the present study, T2 targets sometimes appeared as smeared, horizontally extended images. The horizontal length of the image was about two to three times

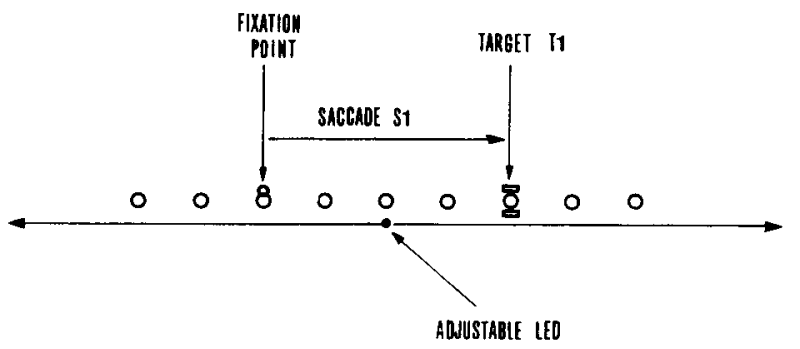

$20 F$

Figure 1. Relative locations of the fixation point, Target T1 for primary Saccade S1, the nine positions of Target $\mathbf{T}$ (large open circles), and the adjustable light spot for perceptual localization.

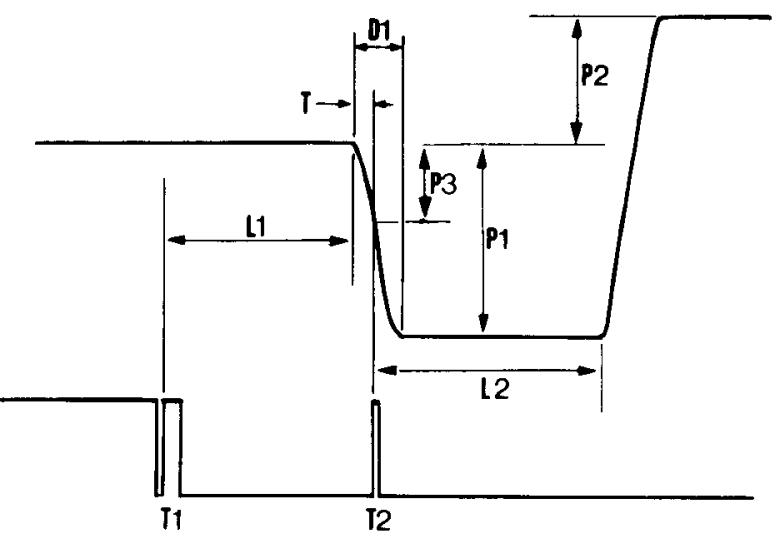

Figure 2. Definition of stimulus and eye movement response parameters. The upper drawing represents eye movements, and the lower drawing represents the time course of the stimuli presentation. See text for definitions of symbols.

$\left(0.6^{\circ}-0.9^{\circ}\right.$ of arc) that of the target's diameter (note that a $2-\mathrm{msec}$ target would leave a path $1^{\circ}$ long if the eye is moving at $500^{\circ} / \mathrm{sec}$ ). In this case, the midpoint of the extended image was regarded as the apparent $T 2$ position. Each subject received 54 experimental trials divided into three daily sessions for 6-8 days. Each session consisted of 18 trials ( 9 positions of Target T2 $\times 2$ repetitions). The interval between the offset of the red LED for perceptual localization and the onset of the warning signal was $2 \mathrm{sec}$. There was a rest period of about $10 \mathrm{~min}$ between sessions.

Five calibration trials were added to each session. Two calibration trials were conducted at the start and the end of each session. The remaining three calibration trials were located after the 4 th, 9th, and 13th trials. In each calibration trial, two LEDs located at $0^{\circ}$ and $+8^{\circ}$ of arc turned on and off in turn repeatedly. The subject made saccades between the two LEDs as accurately as possible.

In addition to the experimental sessions, each subject was given a session of control trials both before and after the series of three experimental sessions. Each control session consisted of 18 trials. In each control trial, Target $\mathrm{T} 1$ was presented for $2 \mathrm{sec}$ at the position of the original fixation point or $8^{\circ}$ to the right of the fixation point. The subject kept watching Target $\mathrm{T} 1$, and $2 \mathrm{msec}$ after the offset of Target T1, Target T2 was presented for $2 \mathrm{msec}$ at one of the nine $\mathrm{T} 2$ positions. The subject moved his/her eyes to Target T2, and then performed a perceptual localization task. The control sessions were introduced to examine the subject's ability to localize visual stimuli briefly presented when his/her eyes remained still.

\section{Data Analysis}

Unfiltered output from the photoelectric eye movement monitor was recorded on a magnetic tape using a data recorder (Nihon Kohden RMG-5304), and later analyzed by a high-speed digital storage scope (Iwatsu DS-6121A).

The response measures of the eye movements consisted of the following seven parameters (see Figure 2): L1 = latency of Saccade S1; P1 = amplitude of Saccade S1; $\mathbf{T}=$ time interval between the onset of Saccade $S 1$ and the occurrence of Target $T 2 ; D 1=$ duration of Saccade S1; L2 = latency of Saccade S2, that is, time interval between the onset of Target $T 2$ and the onset of Saccade S2; $\mathbf{P 2}=$ amplitude between the baseline and Saccade $\mathbf{2}$, that is, the position of the eye after Saccade S2; and P3 = position of the eye when Target $\mathrm{T} 2$ was presented.

\section{Subjects}

The author, a laboratory staff member, and 2 university students served as subjects. 
Table 1

Means and Standard Deviations of Amplitudes (P1), Latencies (L1), and Durations (D1) of S1 Saccades

\begin{tabular}{|c|c|c|c|c|c|c|c|}
\hline \multirow[b]{2}{*}{ Subjects } & \multirow[b]{2}{*}{$N$} & \multicolumn{2}{|c|}{ PI (in deg arc) } & \multicolumn{2}{|c|}{ Ll (in msec) } & \multicolumn{2}{|c|}{ D1 (in msec) } \\
\hline & & $M$ & $S D$ & $M$ & $S D$ & $M$ & $S D$ \\
\hline H.H. & 293 & 89 & 14 & 1680 & 193 & 28.6 & 3.2 \\
\hline E.T. & 21 & 7.9 & & 18 & & & 2.7 \\
\hline S.A. & 167 & 8.7 & 1. & 192.5 & 64 & 33.2 & 4.9 \\
\hline K.S. & 209 & 8.3 & 1.1 & 173.8 & 32.1 & 28.7 & 2.5 \\
\hline
\end{tabular}

Note- $N=$ number of trials.

\section{RESULTS}

\section{Summarizing Remarks}

Subjects E.T. and S.A. each took part in a total of 540 trials (324 experimental and 216 control trials) on 6 separate days. Subject H.H. underwent 720 trials (432 experimental and 288 control trials) on 8 separate days, and Subject K.S. participated in 630 trials (378 experimental and 252 control trials) on 8 separate days. Trials on the first day were practice trials and were excluded from data analysis.

No subject showed S1 saccades with a corrective saccade. However, Subjects K.S. and E.T. sometimes moved their eyes to Target $\mathrm{T} 1$ with two successive saccades. These trials were not used for the data analysis. In addition, data were excluded from the analysis if (1) Saccade S1 occurred before the onset of Target T1 or $400 \mathrm{msec}$ after the offset of the fixation point, (2) the subject did not detect Target T1, or (3) eye movement recording was contaminated by eye blinking. As a result, the data analysis was conducted on 293 trials $(77.5 \%)$, 213 trials $(78.8 \%), 167$ trials $(61.8 \%)$, and 209 trials (64.5\%) of the experimental sessions for Subjects H.H., E.T., S.A., and K.S., respectively.

Table 1 shows the means of amplitudes (P1), latencies (L1), and durations (D1) of S1 saccades. In 2 subjects (H.H. and S.A.), Saccade S1 slightly overshot the position of Target $\mathrm{T} 1\left(8^{\circ}\right.$ of arc).

The subjects made single or double saccades to Target T2. Double saccades were evoked when the position of Target T2 was relatively far from that of Target T1 $\left(+8^{\circ}\right.$ of arc). In this case, the subjects made an additional saccade of normal latency following Saccade S2. When the T2 targets were presented near the position of Target T1, Saccade $\mathbf{S} 2$ did not occur; the subjects made only the $\mathrm{S} 1$ saccade. A large difference between subjects was shown in the latency of Saccade S2. The mean latency of Saccade S2 was $223.7 \mathrm{msec}(S D=65.2), 221.5 \mathrm{msec}$ $(S D=58.4)$, and $428.0 \mathrm{msec}(S D=194.2)$ for Subjects H.H., E.T., and S.A., respectively. The latencies of Saccade S2 obtained from Subject K.S. were extraordinarily large, usually more than $400 \mathrm{msec}$. Therefore, his data were not used for the data analysis of the S2 saccades and oculomotor localization.

Figure 3 shows the latency of Saccade S2 as a function of the size of the saccade. The latency of Saccade S2 increased as the size of the saccade decreased. According to statistical analysis, the effect of the size of the saccade was significant for all subjects [Subject
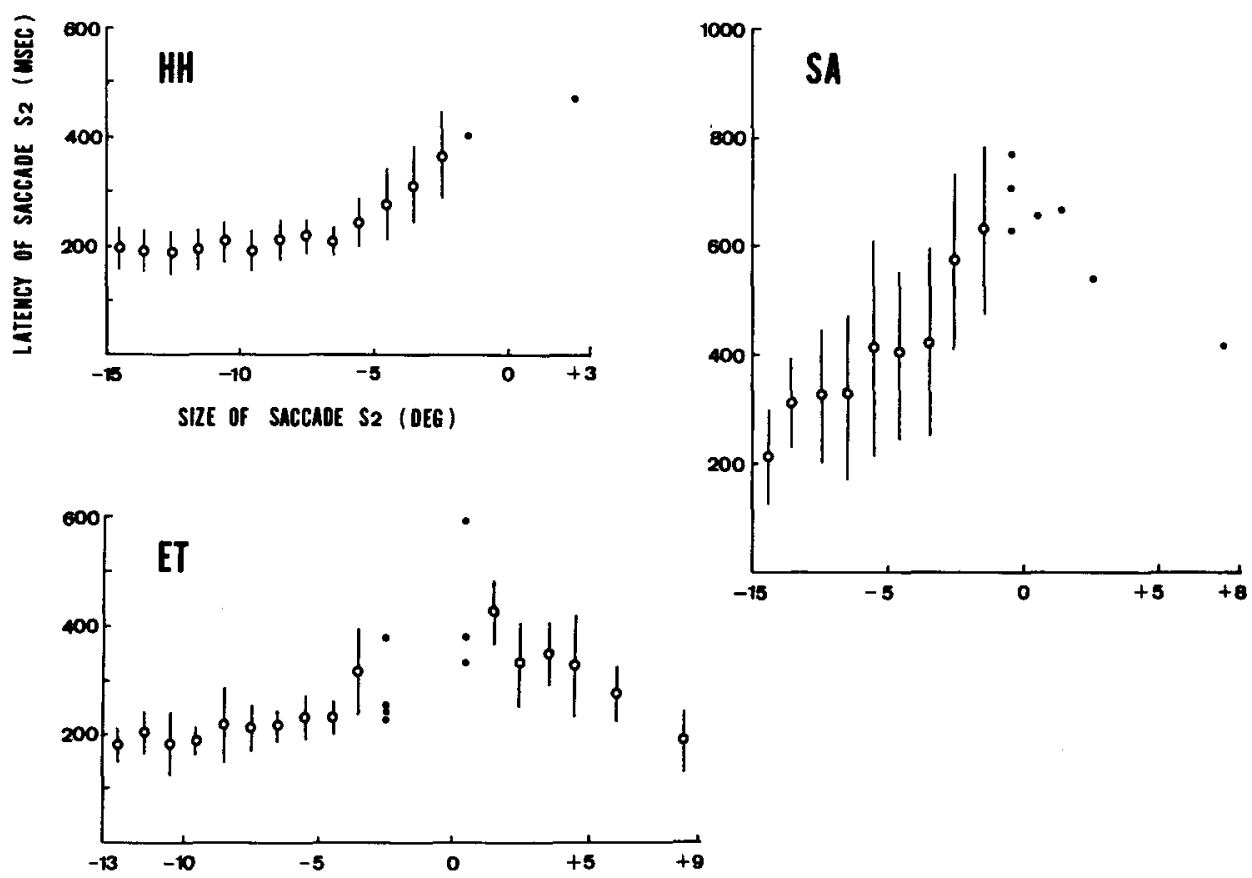

Figure 3. Latency (L2) as a function of size of Saccade S2 for Subjects H.H., E.T., and S.A. Small dots indicate latencies in individual trials that were not used for calculating the mean and the standard deviation because the number of samples was too small. Minus sign in the horizontal axis indicates Saccade S2 in the direction opposite Saccade S1. 
H.H., $F(12,196)=25.85$; Subject E.T., $F(15,132)=$ 14.76; Subject S.A., $F(8,88)=5.65 ; p<.01$ in all cases].

\section{Perceptual Localization}

In Figure 4, the perceptually judged position of Target $T 2$ presented during Saccade $S 1$ is shown as a func- tion of actual T2 position, for each separate subject. There was a difference between subjects in localization performance. Subject H.H. mislocated the T2 targets in the direction opposite Saccade S1 (i.e., to the left side of the actual Target T2 position). On the other hand, Subjects E.T. and K.S. perceptually located the T2 targets near the actual positions.
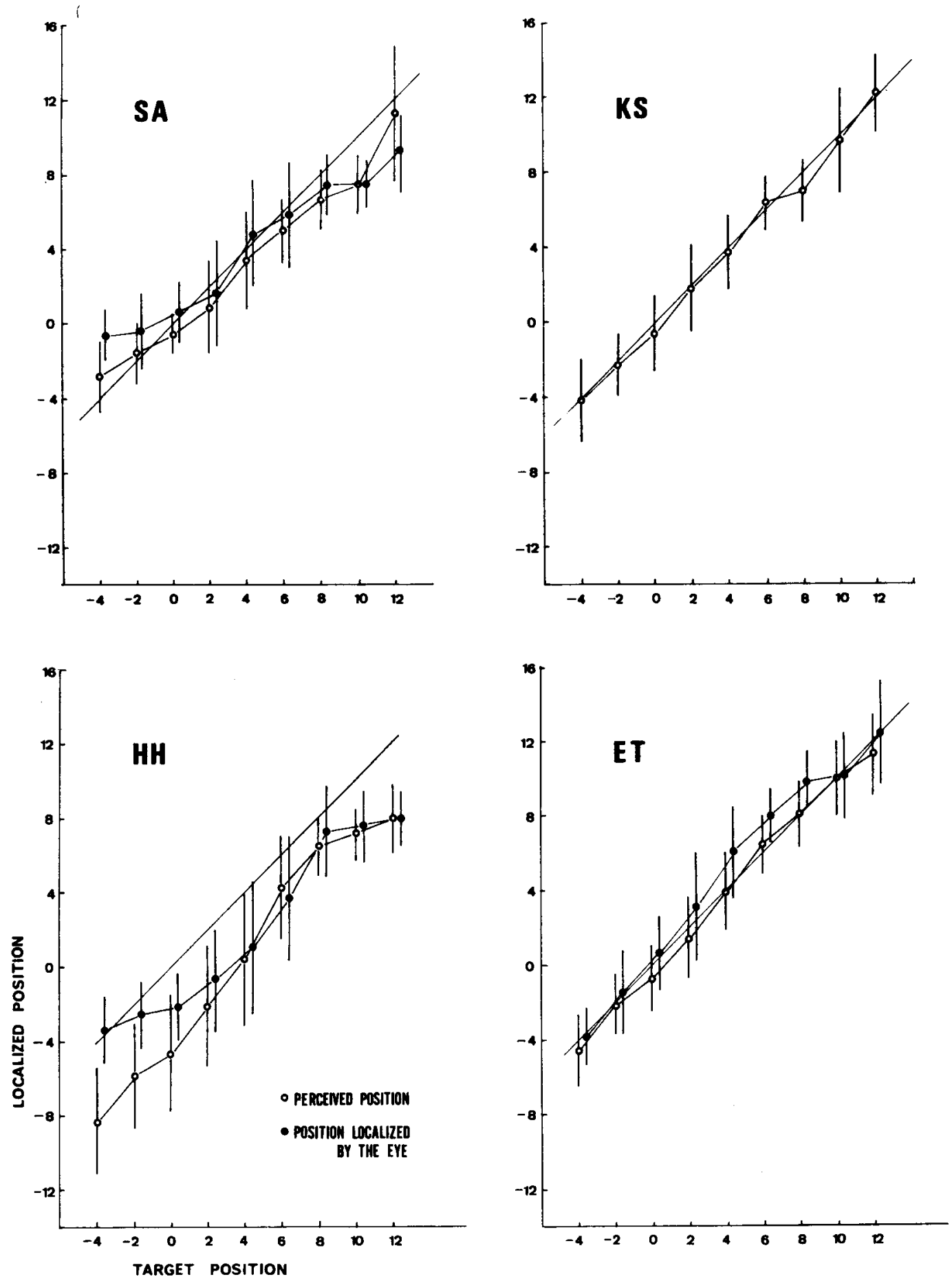

Figure 4. Perceptually localized and oculomotor localized position as a function of actual Target T2 position. Vertical bars indicate the standard deviation. 
Figure 5 shows the error in perceptual localization as a function of $T$ (time interval between the onset of Saccade $S 1$ and the occurrence of Target T2), irrespective of the nine different Target T2 positions. At the beginning of the saccade, all subjects except Subject H.H mislocated the T2 targets in the saccade direction (i.e., to the right side of the actual position). About midway through the saccade, the subjects perceptually located $T 2$ targets near the actual positions. At the end of the saccade, however, the perceptually judged position largely deviated from the actual position in the direction opposite the saccade (i.e., to the left of the actual target position). Statistical analysis showed that the effect of $T$ on perceptual localization was significant for all subjects [Subject E.T., $F(9,199)=26.42$; Subject K.S., $F(12,192)=12.55$; Subject S.A., $F(9,142)=6.65$; Subject H.H., $F(11,271)=29.48 ; p<.01$ in all cases]. The open circles in Figure 5 indicate the perceptually judged positions of Target $T 2$ in control trials. The subjects mislocated $\mathrm{T} 2$ targets especially when $\mathrm{T} 2$ targets were projected to the peripheral retina of the stationary eyes; they judged the $T 2$ targets to be farther from the fixating position $\left(0^{\circ}\right.$ or $+8^{\circ}$ of arc) than the actual target positions. In addition, the size of mislocalization increased with the eccentricity of the T2 positions. However, the size of mislocalization in control trials was clearly smaller than that shown in experimental trials.

The perceptual localization of T2 targets depended on both the actual position of the target in space and its position in a retinotopic coordinate system. I also analyzed the relation between the retinal position of Target $T 2$ and its perceived position. Since the 4 subjects showed similar results, only one typical result (Subject E.T.) is shown in Figure 6, in which perceived position of T2 targets was plotted as a function of retinotopic position. The perceived position largely depended upon the retinal position. A dotted line in Figure 6 is a best fitting regression line. If the perceived position $(Y)$ is completely determined by the retinal position $(X)$, the regression line is expected to be $Y=X$; however, this was not the case.
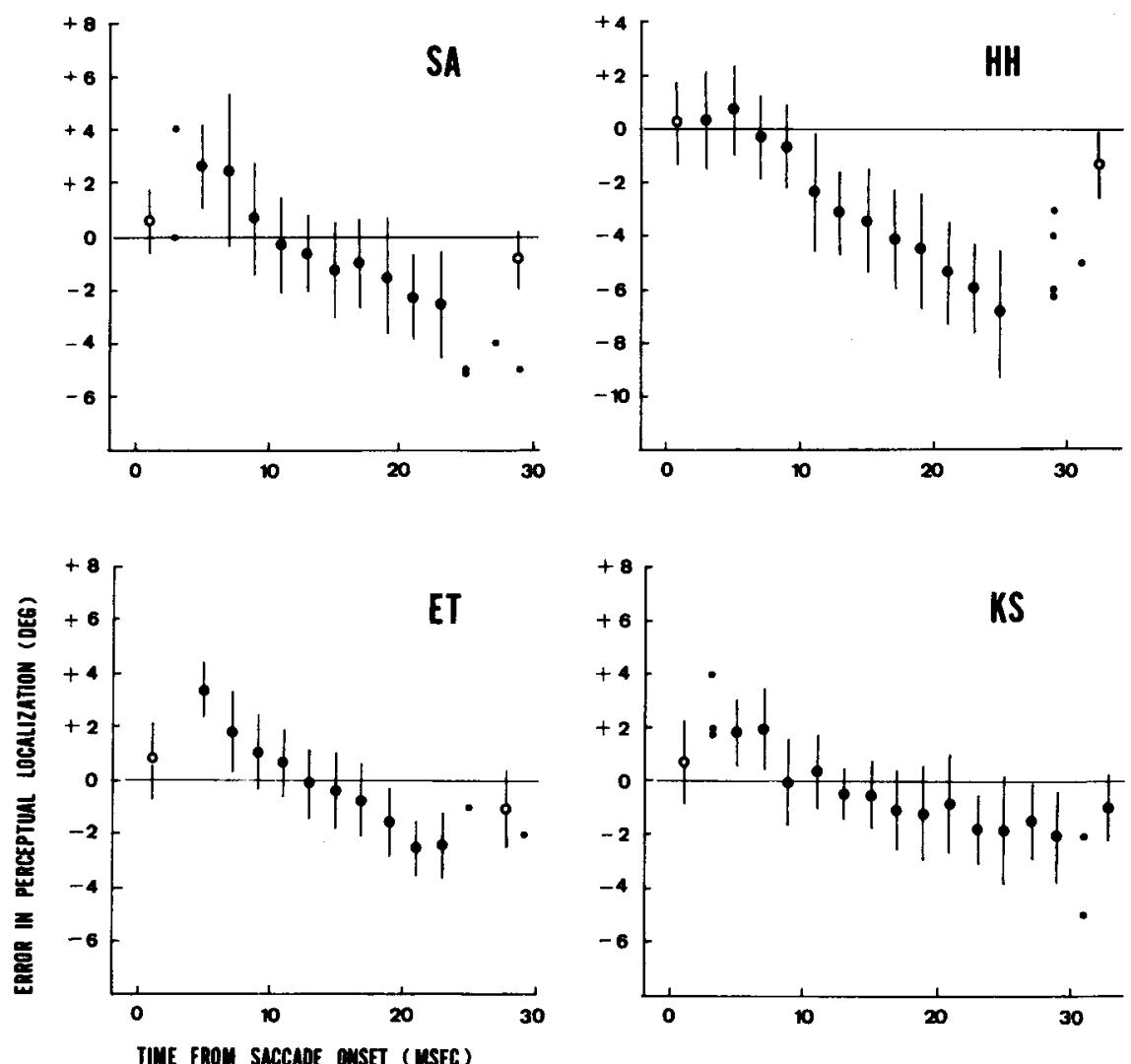

Figure 5. Error in perceptual localization as a function of time interval $(T)$ between the onset of Saccade S1 and the occurrence of Target T2. The vertical axis is the size of the constant error in perceptual localization. The plus sign indicates errors in the direction of Saccade S1, and the minus sign indicates errors in the direction opposite Saceade S1. Open circles show the errors on control trials in which the subject kept watching the position of the fixation point (left circle) or $8^{\circ}$ right of the fixation point (right circle). Vertical bars indicate the standard deviation. Small dots show the error in individual trials that were not used for calculating the mean and the standard deviation because the number of samples was too small. 


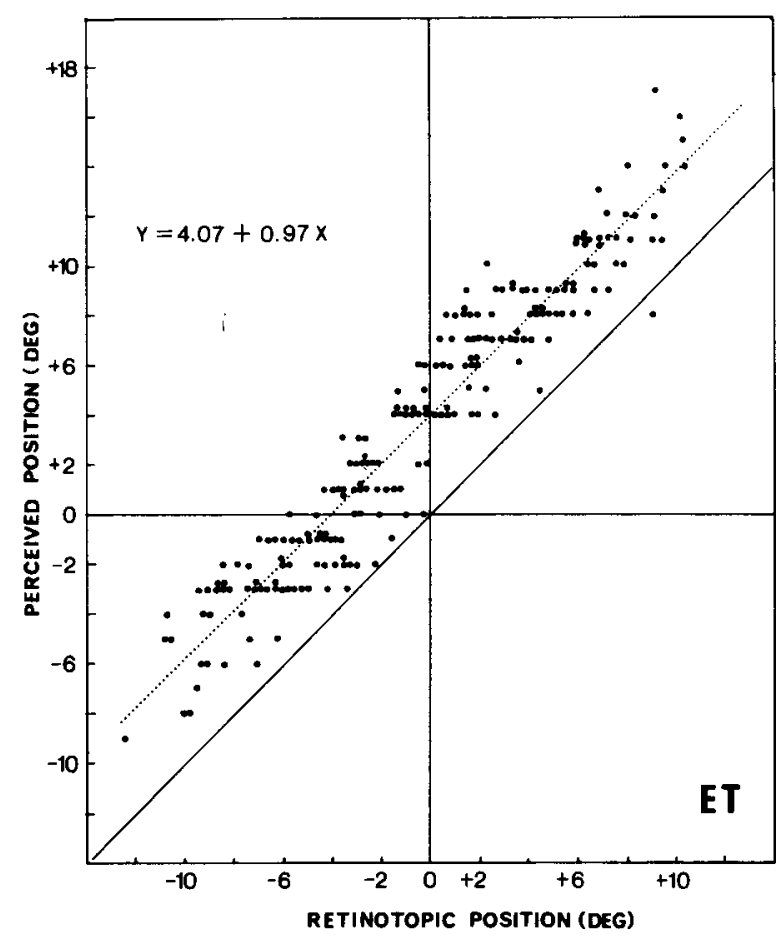

Figure 6. Perceived position of Target $T 2$ as a function of its retinotopic position, that is, the position of Target T2's image on the retina during Saccade S1 (Subject E.T.).

The regression lines obtained from the 4 subjects were as follows:

$$
\begin{array}{ll}
Y=4.07+0.97 X & \text { (Subject E.T.) } \\
Y=4.46+0.94 X & \text { (Subject K.S.) } \\
Y=3.36+0.85 X & \text { (Subject S.A.) } \\
Y=2.11+1.10 X & \text { (Subject H.H.) }
\end{array}
$$

Thus, in all subjects, the slope was nearly 1 , but the intercept varied from subject to subject. This means that at all retinal positions the perceived position shifted about $2^{\circ}$ to $4.5^{\circ}$ toward the saccade direction.

The next analysis, however, showed a more complicated relation between the perceived position and the retinal position. Figure 7 shows errors in perceptual localization as a function of retinal position. The errors were calculated by subtracting the actual position of Target $\mathrm{T} 2$ from its perceived position. Dotted lines in Figure 7 are regression lines calculated for each actual target position. As shown in Figure 7, many slopes of the regression lines were less than 1 ; the mean of the slope was 0.68 , and the range was 0.5 to 1.04 . Similar results were obtained from the remaining 3 subjects.

Figure 7 indicates that an important determinant of the perceived position was the relative retinal position with respect to the actual position. The relative retinotopic position is the distance on the retina between the target image when the target was presented before the onset of Saccade S1 and that when it was presented during Saccade $S 1$. I therefore analyzed the relation between errors in perceptual localization and the relative retinal position (Figure 8). The relative retinal position is calculated by subtracting the actual target position from the retinal position. For example, if a T2 target of $+2^{\circ}$ is presented when the subject's eye has moved $7^{\circ}$ to the right, the retinal position is $-5^{\circ}$, and its relative retinal position is $-7^{\circ}$. Thus, if the minus sign of the relative retinal position is changed to plus, it is equivalent to the eye position at the time when Target $\mathrm{T} 2$ was presented during a saccade. Because, in the present study, subjects always made Saccade $S 1$ to the right, the relative retinal position was less than 0 .

Figure 8 shows the best fitting regression lines of the error in perceptual localization in the relative retinal po-

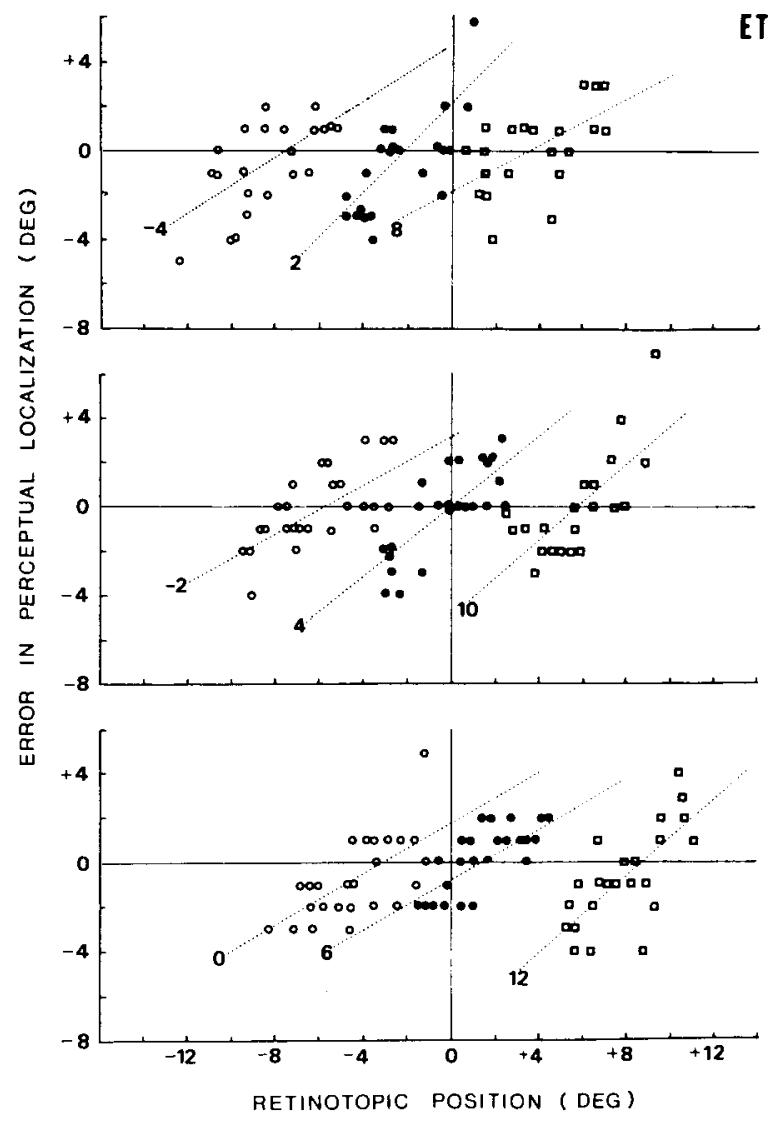

Figure 7. Error in perceptual localization as a function of retinotopic position of Target T2. Plus and minus signs indicate mislocalization to the right (in the saccade direction) and to the left of the actual target position, respectively. Dotted lines are regression lines calculated separately for the nine target positions. Data points obtained for each actual target position are designated by different symbols: $\circ$, target positions $-4^{\circ},-2^{\circ}$, and $0^{\circ} ; \bullet$, target positions $2^{\circ}, 4^{\circ}$, and $6^{\circ} ; 0$, target positions $8^{\circ}, 10^{\circ}$, and $12^{\circ}$. 


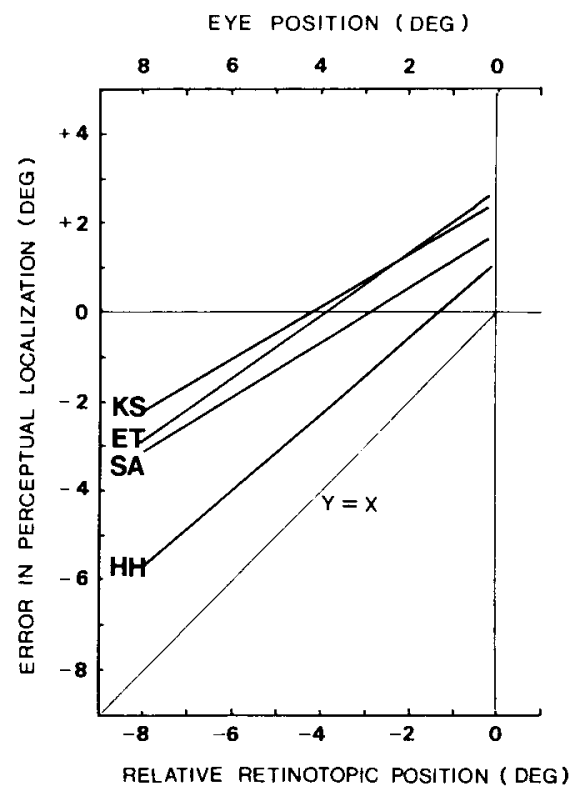

Figure 8. Linear regression of error in perceptual localization $(Y)$ on relative retinotopic position $(X)$. The relative retinotopic position is the distance on the reting between the target image when it was presented before the onset of Saccade S1 and that when the target was presented during Saccade S1.

sition. The regression lines obtained from the 4 subjects were as follows:

$$
\begin{array}{ll}
Y=2.76+0.70 X & \text { (Subject E.T.), } \\
Y=2.57+0.59 X & \text { (Subject K.S.), } \\
Y=1.87+0.62 X & \text { (Subject S.A.), } \\
Y=1.12+0.85 X & \text { (Subject H.H.), }
\end{array}
$$

where $Y$ is the perceived error, and $X$ is the relative retinal position. In all subjects, the slope of the regression line was less than 1 . If the perceived position of Target $T 2$ is completely determined by its retinal position, the regression line is equivalent to the diagonal line: $Y=X$. On the other hand, if the subject's judgment is correct, the regression line is equivalent to the horizontal line: $Y=0$. The results in Figure 8 suggest that the perceived position depended not only on the retinal position, but also on the actual target position.

Finally, therefore, I examined the interaction of the two determinants of the perceived localization, that is, the retinal and the actual positions of the target. For this purpose, we computed a multiregression function by using the retinal and the actual positions as two independent variables. The functions obtained from the 4 subjects were as follows:

$$
\begin{array}{ll}
Y=2.57+0.68 X_{1}+0.34 X_{2}, R=0.96 & \text { (Subject E.T.) } \\
Y=2.46+0.57 X_{1}+0.43 X_{2}, R=0.95 & \text { (Subject K.S.) } \\
Y=2.82+0.71 X_{1}+0.13 X_{2}, R=0.93 & \text { (Subject S.A.) } \\
Y=0.71+0.86 X_{1}+0.27 X_{2}, R=0.96 & \text { (Subject H.H.) }
\end{array}
$$

where $Y$ is perceived position, $X_{1}$ is retinal position, $X_{2}$ is actual position of the Target $\mathrm{T} 2$, and $R$ is the multiple correlation coefficient of regression. In all subjects, the regression coefficient of retinal position $\left(X_{1}\right)$ was larger than that of actual position $\left(X_{2}\right)$. This means that the perceived position of Target $\mathrm{T} 2$ was primarily determined by its retinal position. The heavy dependence of the perceived position of Target $T 2$ on its retinal position is very close to the results for small saccades reported by Matin et al. (1969).

\section{Oculomotor Localization}

Filled circles in Figure 4 show positions localized by the eye as a function of actual position of Target $\mathrm{T} 2$. Because Subject K.S. showed extremely long latencies of Saccade S2 (usually more than $\mathbf{4 0 0} \mathrm{msec}$ ), his data were excluded from the following analysis. Although in Subjects H.H. and S.A. there were distinct differences in localized position between the two different localization tasks (i.e., perceptual and oculomotor), it is unclear which of the two tasks is more accurate in localizing actual positions of Target T2. Actually, it seems that the results of the two kinds of localization were very similar. In addition, as in perceptual localization, the subjects showed large variance in oculomotor localization.

To examine the effect of retinal position of Target $\mathrm{T} 2$ on its oculomotor localization, we calculated the linear regression function of the oculomotor localization on the retinal position of the target. The slope (regression coefficient) of the linear regression function was $0.98,0.64$, and 0.78 for Subject E.T., S.A., and H.H., respectively. In 2 subjects, S.A. and H.H., the slope was smaller than that obtained for perceptual localization. However, as in perceptual localization, subsequent analysis showed that the relation between the localized position of Target T2 and its retinal position was complicated.

Figure 9 shows regression lines of error in oculomotor localization on the retinotopic position of the target, obtained separately for each actual target position. In this subject (H.H.), except for the actual positions of $2^{\circ}, 4^{\circ}$, and $6^{\circ}$, the regression coefficient was small: the mean of the regression coefficients was 0.28 . In addition, at the target position of $-4^{\circ}$, the regression coefficient was not significantly different from 0 [regression coefficient $=$ $0.14, F(1,30)=1.89]$. Similar results were obtained in the remaining 2 subjects. In these subjects, the regression coefficient was significantly different from 0 in only five (Subject E.T.) and two (Subject S.A.) target positions. This suggests that in oculomotor localization the role of retinal position is less significant than in perceptual localization.

Next, I computed a multiregression function for oculomotor localization. The functions obtained from the 3 subjects were as follows:

$$
\begin{array}{ll}
Y=2.46+0.37 X_{1}+0.71 X_{2}, R=0.94 & \text { (Subject E.T.), } \\
Y=2.80+0.36 X_{1}+0.31 X_{2}, R=0.85 & \text { (Subject S.A.), } \\
Y=1.49+0.51 X_{1}+0.31 X_{2}, R=0.88 & \text { (Subject H.H.), }
\end{array}
$$




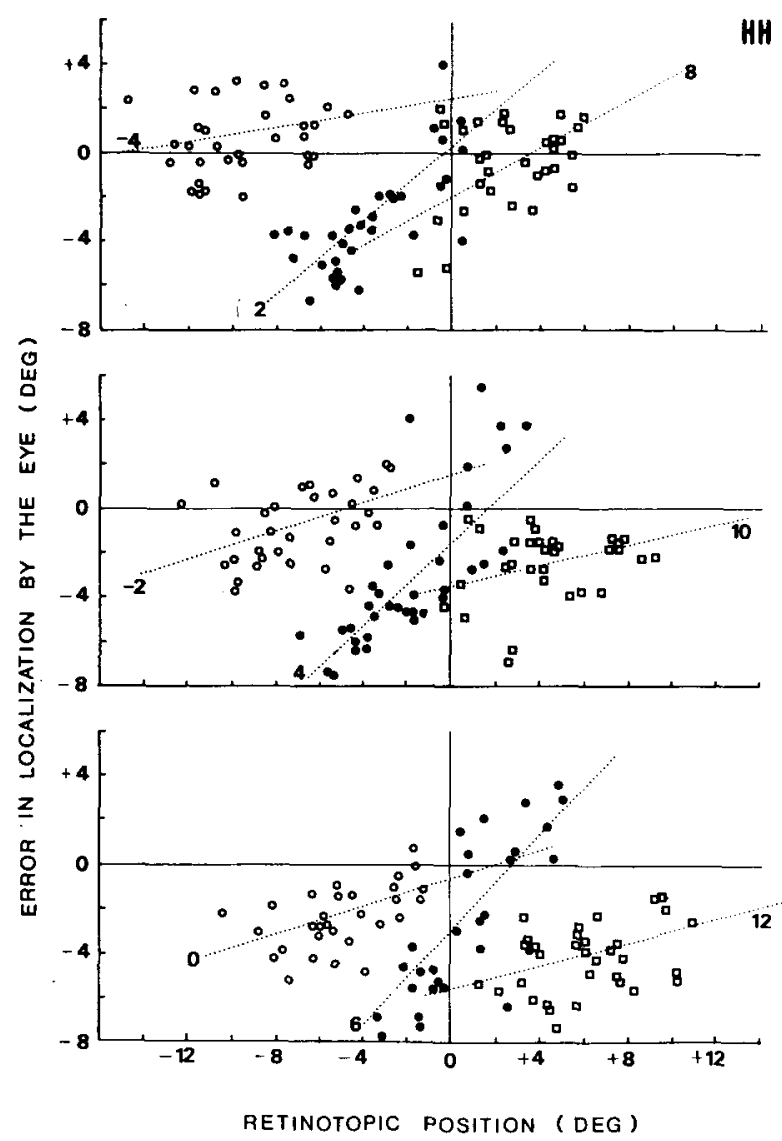

Figure 9. Error in localization by the eye as a function of retinotopic position of Target T2 (Subject H.H.). Dotted lines are regression lines calculated separately for the nine target positions. Data points are designated by different symbols according to their actual target positions.

where $Y$ is position localized by the eye, $X_{1}$ is retinal position, $X_{2}$ is actual position of Target $\mathrm{T} 2$, and $R$ is the multiple correlation coefficient of regression. In all subjects, the size of the regression coefficient of the retinal position $\left(X_{1}\right)$ was smaller than that of the regression function obtained for perceptual localization.

\section{Relation Between Two Types of Localization}

As described above, the results of multiregression analysis suggest that the retinal and the actual positions of Target T2 differentially influenced the two types of localization. Perceptual localization was more influenced by the retinal position of the target than by its actual position. On the other hand, oculomotor localization was more influenced by the actual position of the target. As shown in Figure 4, however, this does not mean that subjects localized the target more accurately by the eye than by perceptual judgment.

Figure 10 shows the relation between oculomotor and perceptually localized positions. In Subject H.H., a large discrepancy between the two kinds of localization occurred when Target T2 was seen on the left of the origi- nal fixation point. In many of these trials, the eye did not reach the perceived target position. On the other hand, Subject S.A. showed a large discrepancy at perceived positions of $10^{\circ}$ to $17^{\circ}$, in which the subject made only a primary Saccade S1. Here, again, the eye did not reach the perceived T2 position. In Subject E.T., oculomotor localization without Saccade S2 was shown at a wide range of perceived positions (from $4^{\circ}$ to $12^{\circ}$ ). Therefore, the discrepancy between the two kinds of localization seems to be caused by the imprecise localization ability of the oculomotor system.

\section{DISCUSSION}

\section{Perceptual Localization During Saccade}

In the present experiment, subjects perceptually mislocated the visual stimulus briefly presented during a saccade. The localization error depended on the time between saccade onset and the occurrence of the visual stimulus. At the beginning of a saccade visual stimuli were mislocated in the saccade direction, whereas at the end of the saccade they were mislocated in the opposite direction from the saccade.

Perceptual mislocalization also was observed in nonsaccade control trials. In control trials, the subjects judged the visual stimuli presented at the peripheral retina to be more eccentric than their actual position from the fixating position. Therefore, mislocalization observed in saccade trials can, in part, be explained in terms of indigenous peripheral-central differences in localization ability of the visual system. However, it is evident that the errors in saccade trials were larger than what would be predicted from the results of nonsaccade control trials.

The regression lines shown in Figure 8 indicate that perceptual localization was not completely determined by the retinal position of a target. First, the regression lines were not equivalent to the function of $Y=X$. Instead, the intercept of the regression lines was larger than 0 . In other words, subjects perceptually locate Target $T 2$ on the right side of the position expected if the localization was determined only by its retinal position. The shift of localization was shown to be the same at all actual and retinal positions (Figures 6 and 7). However, the intercept of the regression lines varied from subject to subject, indicating that there was a substantial difference in the size of the shift described above. Second, the slope of the regression lines was less than 1 . This means that perceptual localization depended not only on the retinal position of Target T2, but also on its actual position. The results of multiregression analysis show that in all subjects the regression coefficient of retinal position was larger than that of its actual position, indicating that the retinal position was a more important determinant of perceptual localization than was the actual position.

According to the model of visual localization proposed by von Holst and Mittelstaedt (1950) and others, information derived from nonretinal sources (extraretinal signals) and information about the position of the target's 

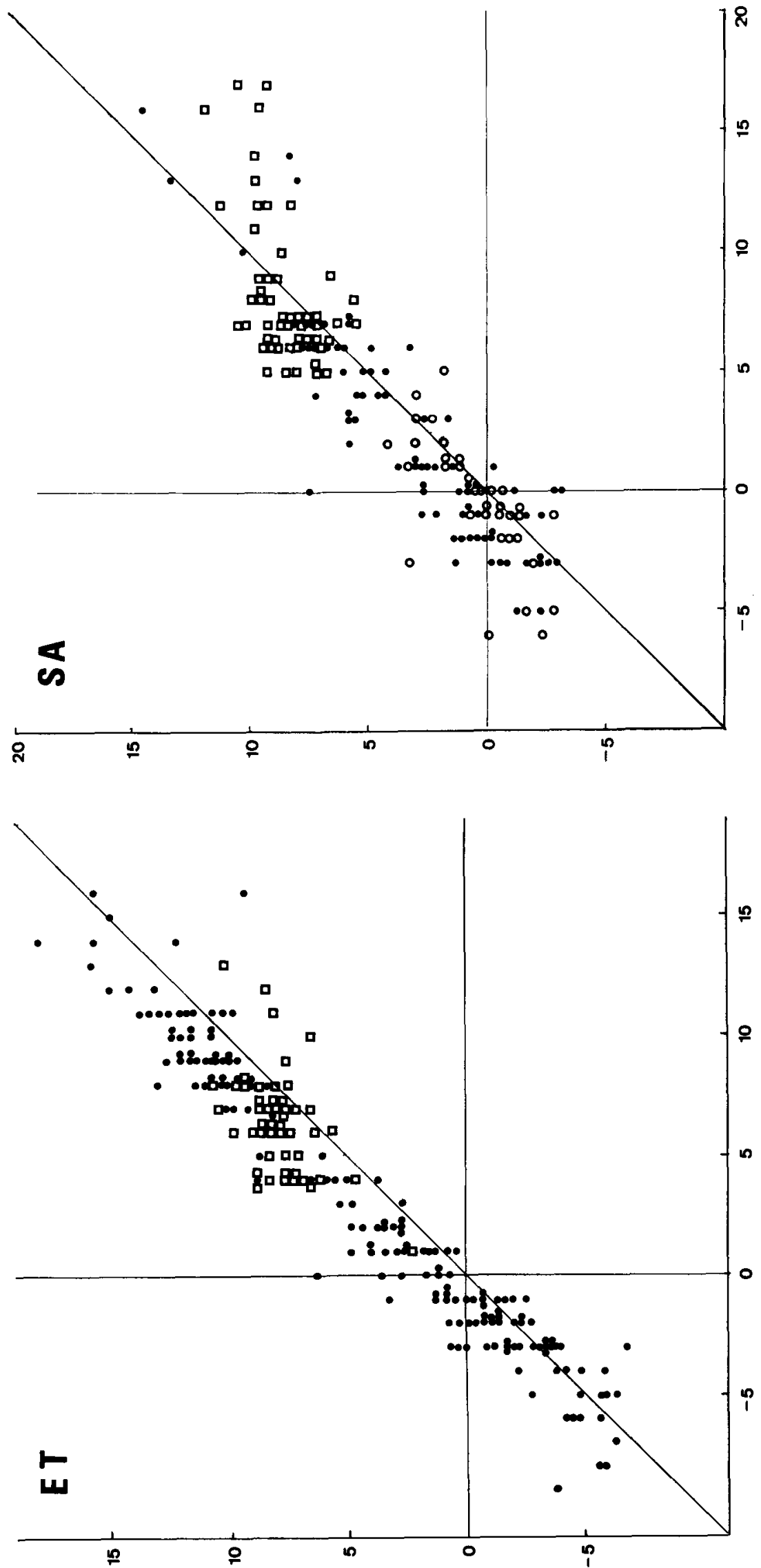


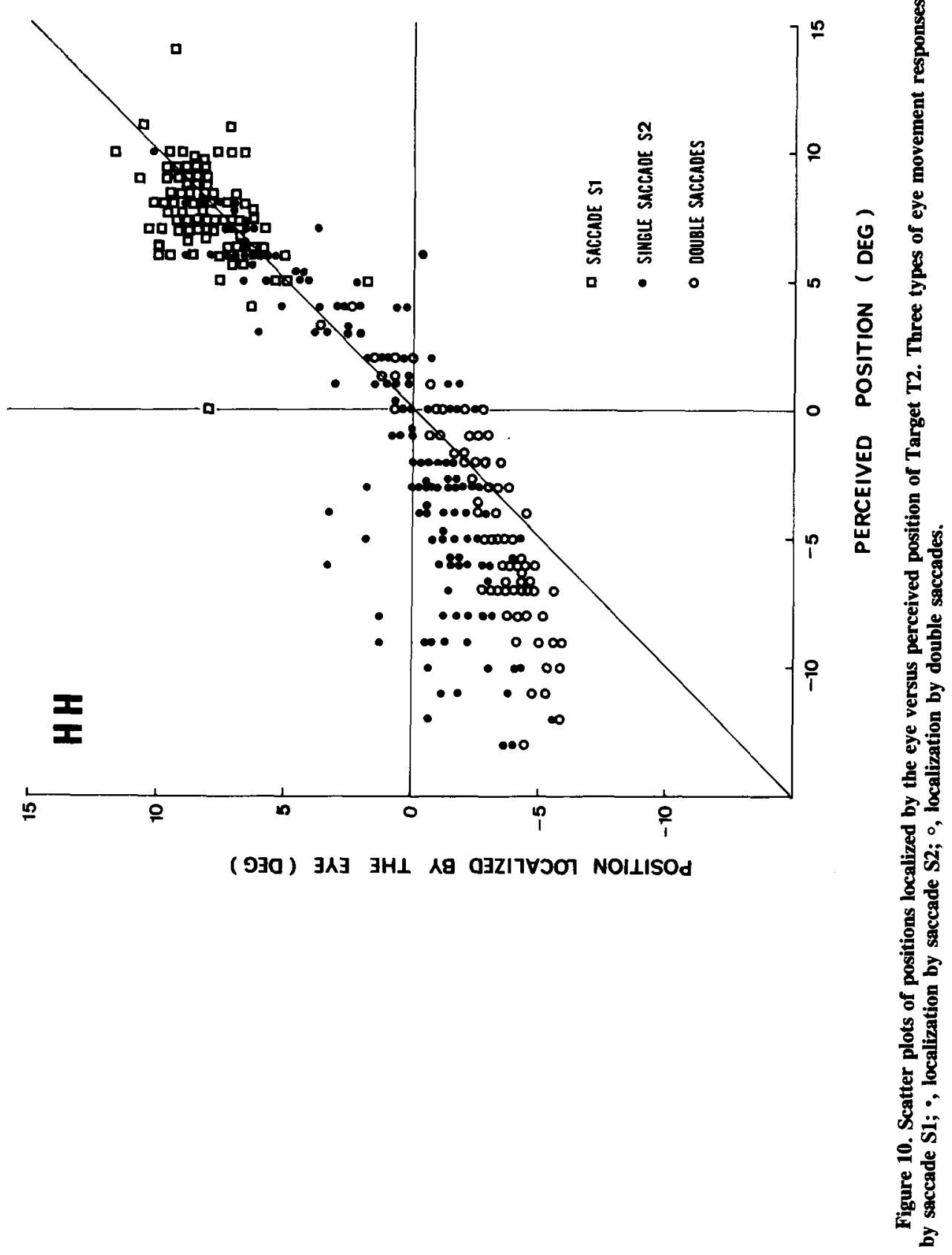


retinal image are added to determine the accurate target position. If retinal errors are completely compensated by extraretinal information, the perceptually judged position agrees with actual target position. This was not the case in the present study. Perceptual localization was primarily determined by the retinal locus of the target's image. However, this does not mean that extraretinal signals played no role in perceptual localization during a saccade. The fact that the slope of regression lines shown in Figure 8 was not 1 suggests that subjects to some degree used extraretinal signals in their perceptual localization. Matin (1976) stated that

It is not true that a canceling extraretinal signal shifts the relation of stimulated retinal locus and perceived visual direction at a rate that parallels the actual saccadic eye movement. ... It begins before the saccade, and continues to change for a considerable period after the saccade. (pp. 209-211)

This conclusion agrees with the result of the present study, becuase the results shown in Figures 5 and 8 suggest that, at the beginning of a saccade, overcompensation by nonretinal information occurred, resulting in mislocation in the saccade direction, whereas at the end of a saccade, undercompensation resulting in mislocation in the opposite direction from the saccade. (Note that in Figure 8 the absolute value of the relative retinal position is equivalent to the position of the eye during a saccade.)

However, this interpretation seems incomplete. First, Figure 8 indicates that the subjects' perceptual localization was determined primarily by the retinal position of the target, and that at all eye positions during a saccade (relative retinal position), the perceptually judged position shifted to the right (i.e., in the saccade direction) from the position that would be predicted if the perceptual localization was determined only by the retinal position of the target. Moreover, the size of the shift (i.e., the intercept of the regression lines) was different among subjects. In Subject H.H., the size of the shift was small. As a result, he showed only a small mislocation at the beginning of a saccade. On the other hand, the remaining 3 subjects showed a large shift, resulting in a large mislocation at the beginning of a saccade. Second, the slope of the regression lines varied from subject to subject. Interestingly, the slope decreased as the intercept increased. In Subject H.H., the intercept was small, and the slope was close to 1 . This means that Subject H.H. judged the target position mainly by its retinal position. On the other hand, in the remaining 3 subjects, the intercept was large, and the slope was approximately 0.6 to 0.7 ; that is, in these subjects, the localized position deviated toward the saccade direction from the retinotopic position of the target.

What do the slope and the intercept of the regression lines mean? I speculate that these reflect two different kinds of extraretinal information that were used by sub- jects in perceptual localization. First, in this experiment, the position of a saccade goal (Target T1) was constant. Therefore, the size of Saccade S1 was about the same in all trials. However, because the subjects could not know beforehand the exact time of Target $T 2$ presentation, the best strategy was to postulate that the targets would be presented midway through the saccades. The intercept of the regression lines, I think, shows this kind of "presaccade" information. Second, subjects could, to some degree, know the eye position in the orbit when a target was presented. This kind of "during-saccade" information is the so-called extraretinal eye position information that has been postulated by many earlier researchers. I think that the slope of the regression lines shows this kind of extraretinal information. If the during-saccade extraretinal information is complete, the slope will become 0 . The results of the present study suggest that the ability to use the pre- and during-saccade information differed among the subjects.

\section{Oculomotor Localization}

The results of multiregression analysis show that in oculomotor localization the role of retinal position was less significant than it was in perceptual localization. However, this does not mean that subjects more accurately located the target in oculomotor localization than they did in perceptual localization. Detailed analysis of the relation between the two measures (Figure 10) suggests that a large discrepancy between the two measures was due to the imprecise localization ability of the oculomotor system. It has been well known that saccades sometimes undershoot the actual target position (Becker \& Fuchs, 1969; Weber \& Daroff, 1971). When a target was extinguished at the time of saccade onset, the possibility of occurrence of a secondary, corrective saccade was low (Prablanc \& Jeannerod, 1975). These earlier findings agree with the oculomotor localization found in the present study. That is, in the present study, Target $\mathrm{T} 2$ for the localization task was presented for only $2 \mathrm{msec}$ during a prior primary saccade, and therefore was extingished before the onset of the second saccade. The second saccades were never followed by a corrective saccade, and on many trials did not reach the perceived target position. Moreover, when the target was seen near Target $\mathrm{T} 1$, subjects sometimes made only one saccade (S1), and did not make a secondary saccade (S2). This is additional evidence for the imprecise localization ability of the oculomotor system.

Hallett and Lightstone (1976b) reported that a fixation target flashed during a saccade elicited a subsequent goaldirected saccade of normal amplitude. In the present study, however, the eye moved not to the actual target position, but to its apparent position. This does not agree with Hallett and Lightstone's finding. One reason for this discrepancy is that in Hallett and Lightstone's experiment, the flash always occurred at the same time during the saccade, and after each secondary saccade the position of the 
flash was revealed to the subjects. Through many trials and with the knowledge of actual results, subjects might have learned to make the appropriate secondary saccade.

\section{Other Findings}

Latencies (L2s) of Saccade S2 largely depended on the apparent position of Target $\mathrm{T} 2$ and, therefore, on the size of Saccade S2 (Figure 3). In Subjects H.H. and E.T., for example, L2 varied from about $200 \mathrm{msec}$ (when the size of Saccade S2 was more than $5^{\circ}$ ) to $400 \mathrm{msec}$ (when the size of Saccade S2 was $2^{\circ}$ to $3^{\circ}$ ). This variance in L2 was very large in comparison with that reported in earlier studies that examined the relation between saccade latency and retinal eccentricity of targets. Except when retinal eccentricity is extremely small (less than $0.5^{\circ}$ ) or large (more than $20^{\circ}$ ), saccade latency is not affected by retinal eccentricity (Findlay, 1985). Since in the present study T2 targets were presented during a saccade, saccade size did not correspond to retinal eccentricity of the targets. Therefore, it is impossible to directly compare L2s of the present study with saccade latencies studied in relation to retinal eccentricity of targets. The variance of $L 2$ shown in the present study was, in any event, too large.

Hallett and Lightstone (1976a) stated that whatever the duration $\Delta$ (duration of visual target presented during a saccade), saccades that occurred in the dark had essentially normal latencies. This was also the case in the present study when the saccade amplitude was larger than about $5^{\circ}$ of arc, but not when the saccade amplitude was small. The long L2 shown in the present study seems to be due to the following facts. First, in the present experiment subjects sometimes reported smearing of the Target $\mathrm{T} 2$ image. In addition, $\mathrm{T} 2$ targets sometimes appeared as horizontally extended images with a horizontal length two to three times that of the actual target's diameter. In these trials, the subjects had difficulty in determining the apparent position of Target T2. Second, the oculomotor system showed only an imprecise localization ability. When the $\mathrm{T} 2$ targets were presented near the $\mathrm{T} 1$ position, the subjects frequently omitted the $S 2$ saccades; they made only S1 saccades. Figure 10 shows that omission of S2 saccades occurred when Target $\mathrm{T} 2$ appeared within about $4^{\circ}$ of arc around the T1 position. Thus, I speculate that in these cases, the subjects sometimes had difficulty in deciding whether they should make an S2 saccade or not.

\section{CONCLUSIONS}

The results of this study have shown that, when a visual target is flashed during a saccade in the dark, the size of error in perceptual judgment of the target position is a time function. Targets flashed at the beginning of the saccade were mislocalized in the saccade direction. On the other hand, targets flashed at the end of the saccade were mislocalized in the direction opposite the saccade. I examined the interaction of the two determinants of the per- ceptual localization (i.e., the retinal and the actual position of the target) by computing a multiregression function, and found that the perceived position of the target was primarily determined by its retinal position. In addition, it was also suggested that although subjects to some degree used extraretinal eye position information in their perceptual judgments, the role of that information was rather small. These findings are consistent with the earlier findings (Matin et al., 1969) reported for small saccades.

I compared the target positions localized by eye responses with the perceptually judged positions, and found that the results of the two types of localization were very similar. The results of multiregression analysis suggested that the retinal and the actual positions of targets differentially influenced the two types of localization. Oculomotor localization was more influenced by the actual position of targets. However, this does not mean that the eye moved to the actual target positions. Instead, the eye moved to the perceptually judged positions. This finding does not agree with the results of Hallett and Lightstone (1976a, 1976b). The small discrepancy shown between the two types of localization task is in part explained by the imprecise localization ability of the saccade system.

\section{REFERENCES}

BeCKer, W., \& FuchS, A. F. (1969). Further properties of the human saccadic system: Eye movements and correction saccades with and without visual fixation points. Vision Research, 9, 1247-1258.

BISCHOF, N., \& KRAMER, E. (1968). Untersuchungen und Überlegungen zur Richtungswahrnehmung bei willkürlichen sakkadischen Augenbewegungen. Psychologische Forschung, 32, 185-218.

Bridgeman, B., Lewis, S., Heit, G., \& NAGle, M. (1979). Relation between cognitive and motor-oriented systems of visual position perception. Journal of Experimental Psychology: Human Perception \& Performance, 5, 692-700.

FINDLAY, J. M. (1985). Visual information processing for saccadic eye movements. In A. Hein \& M. Jeannerod (Eds.), Spatially oriented behavior (pp. 281-303). Berlin: Springer-Verlag.

Hallett, P. E., \& Lightstone, A. D. (1976a). Saccadic eye movements towards stimuli triggered by prior saccades. Vision Research, 16, 99-106.

Hallett, P. E., \& Lightstone, A. D. (1976b). Saccadic eye movements to flashed targets. Vision Research, 16, 107-114.

HANSEN, R. M. (1979). Spatial localization during pursuit eye movements. Vision Research, 19, 1213-1221.

Hansen, R. M., Skavenski, A. A. (1977). Accuracy of eye position information for motor control. Vision Research, 17, 919-926.

HANSEN, R. M., \& SkAVENSKI, A. A. (1985). Accuracy of spatial localization near the time of saccadic eye movements. Vision Research, 25, 1077-1082.

HoNDA, H. (1985). Spatial localization in saccade and pursuit-eyemovement conditions: A comparison of perceptual and motor measures. Perception \& Psychophysics, 38, 41-46.

MATEEFF, S. (1978). Saccadic eye movements and localization of visual stimuli. Perception \& Psychophysics, 24, 215-224.

Matin, L. (1976). Saccades and extraretinal signals for visual direction. In A. Monty \& J. W. Senders (Eds.), Eye movements and psychological processes (pp. 205-219). New York: Erlbaum.

Matin, L., Matin, E., \& Pearce, D. G. (1969). Visual perception of direction when voluntary saccades occur: I. Relation of visual direc- 
tion of fixation target extinguished before a saccade to a flash presented during the saccades. Perception \& Psychophysics, 5, 65-80.

Matin, L., Matin, E., \& Pola, J. (1970). Visual perception of direction when voluntary saccades occur: II. Relation of visual direction of a fixation target extinguished before a saccade to a subsequent test flash presented before the saccade. Perception \& Psychophysics, 8 , 9-14.

Matin, L., \& Pearce, D. G. (1965). Visual perception of direction for stimuli flashed during voluntary saccadic eye movements. Science, $148,1485-1488$.

Mitrani, L., Dimitrov, G., Yakimoff, N., \& Mateeff, S. (1979).
Oculomotor and perceptual localization during smooth eye movements. Vision Research, 19, 609-612.

Prablanc, C., \& Jeannerod, M. (1975). Corrective saccades: Dependence on retinal reafference signals. Vision Research, 15, 465-469. von Holst, E., \& MitTelstaedt, H. (1950). Das Reafferenzprinzip. Naturwissenschaften, 37, 464-476.

Weber, R. B., \& DAROFF, R. B. (1971). The metric of horizontal saccadic eye movements in normal humans. Vision Research, 11, 921-928.

(Manuscript received September 1, 1987; revision accepted for publication August 9, 1988.) 